\title{
Desnutrición en prescolares de familias migrantes*
}

Georgina Mayela N úñez-Rocha, M.S.P., ${ }^{(1)}$ Magaly Bullen-N avarro, M.S.P., ${ }^{(2)}$

Blanca Cecilia Castillo-Treviño, M.S.P., ${ }^{(3)}$ Elizabeth Solís-Pérez, M.S.P. ${ }^{(4)}$

\section{Núñez-Rocha GM, Bullen-Navarro $M$, Castillo-Treviño BC, Solís-Pérez E. Desnutrición en prescolares de familias migrantes.} Salud Publica Mex 1998;40:248-255.

\section{Resumen}

Objetivo. Comparar la prevalencia de desnutrición entre prescolares migrantes y no migrantes. Material y métodos. Se seleccionaron al azar 160 infantes de 1-6 años de edad, de parvularios de muy alta marginación. Se excluyeron aquellos con malformaciones congénitas o bajo alguna intervención nutricional. La migración se definió como el desplazamiento geográfico $<6$ años, y la desnutrición, a partir de los indicadores peso/talla y talla/edad recomendados por la 0 rganización Mundial de la Salud. Resultados El $59.4 \%$ de los niños provenía de familias cuyo jefe tenía una ocupación no calificada; $27.5 \%$ de los mismos contaba con primaria incompleta, y $52.8 \%$ refirió migración. La prevalencia de desnutrición fue de $51.3 \%$ en migrantes y de $28.8 \%$ en no migrantes ( $R P=2.6, I C 95 \%=1.2,5.2, p=0.006$ ). Los infantes migrantes presentaron una media de puntaje $Z$ de $-2.4 \pm .40$, mientras que en los no migrantes fue de $-2.3 \pm .33$, según el indicador talla/edad. Conclusiones. La desnutrición crónica en este grupo de riesgo justifica la prioridad de implantar estrategias de intervención alimentaria. Se recomienda considerar la migración en la planeación en salud.

Palabras clave: trastornos de la nutrición infantil; migración rural-urbana; infante; México

\author{
Núñez-Rocha GM, Bullen-Navarro $M$, \\ Castillo-Treviño BC, Solís-Pérez E. \\ Malnutrition in preschool \\ children of migrant families. \\ Salud Publica Mex 1998;40:248-255.
}

\begin{abstract}
A bstract
Objective.To compare malnutrition rates between migrant and non-migrant children. Material and methods. 0 nehundred and sixty children 1-6 years old were selected at random from schools located in highly marginated areas. Excluded were infants with congenital malformations or under nutritional intervention. Migration was defined as any geographical movement during the last 6 years. Malnutrition was assessed through the weight/height and height/ age indicators, as recommended by the W orld Health $\mathrm{O}$ ganization. Results. Fifty-nine percent of the infants belonged to families whose father had a non-qualified occupation, $27.5 \%$ of them did not finish elementary school. Fifty-three referred migration; malnutrition rate was 51.3\% among migrant infants and $28.8 \%$, among non-migrant infants $(O \mathrm{R}=2.6, \mathrm{C} \mid 95 \%=1.2,5.2, \mathrm{p}=0.006)$. Migrant children registered a mean $Z$ score of $-2.4 \pm .40$ and non-migrant children, $-2.3 \pm .33$, based on the indicator height for age. Conclusions Chronic malnutrition among migrant infants justifies a nutritional intervention, they constitute a specific group at risk. Migration should be considered for health planning.
\end{abstract}

Key words: infant nutrition disorders; rural-urban migration; child, preschool; Mexico

* Este proyecto constituye parte de la tesis realizada para obtener el grado de Maestría en Salud Pública, con especialidad en N utrición Comunitaria en la Facultad de Salud Pública y N utrición de la Universidad Autónoma de N uevo León, México.

(1) Investigador A sociado "A", Unidad Regional de Investigación Epidemiológica y en Servicios de Salud. Instituto Mexicano del Seguro Social, Monterrey N uevo León, México.

(2) Jefatura de Control Sanitario de Alimentos y Vigilancia Veterinaria de la Región de la Provincia de Coclé. Ministerio de Salud, República de Panamá.

(3) Docente de la Facultad de Salud Pública y N utrición. Universidad Autónoma de N uevo León, México.

(4) Directora de la Facultad de Salud Pública y Nutrición. Universidad Autónoma de N uevo León, México.

Fecha de recibido: 8 de abril de 1997 • Fecha de aprobado: 16 de diciembre de 1997

Solicitud de sobretiros: Dra. Georgina Mayela N úñez Rocha. Enebro 4317, colonia Residencial Cedros, 64370 Monterrey, N uevo León, México. 
a desnutrición es un problema de gran magnitud en todo el mundo, según los datos epidemiológicos de países en vías de desarrollo. ${ }^{1}$ En México, la última Encuesta Nacional de Nutrición, realizada en 1988, reveló una prevalencia de $37.5 \%$ de desnutrición en niños prescolares, de acuerdo con el indicador talla para la edad con valores menores a -2 desviaciones estándar por debajo del $95 \%$ de la mediana de referencia. ${ }^{2}$ Por otra parte, las estadísticas de la Secretaría de Salud muestran que las deficiencias de la nutrición ocuparon el quinto lugar en México como causa de mortalidad prescolar, con una tasa de 8.5 por 100000 habitantes en $1993 .{ }^{3}$

El área metropolitana de la ciudad de Monterrey tiene una población de más de tres millones de habitantes; se considera que es la capital industrial de México y, por ende, un centro de atracción en el proceso migratorio. El estado de Nuevo León se ha señalado como la entidad del país con mayor movilidad interna: la población migrante estimada es de $37.8 \%{ }^{4}$ La migración como cambio social incide, de manera fundamental, en la salud nutricional de los prescolares; el estilo de vida y sus repercusiones se reflejan en un organismo que se encuentra en proceso activo de crecimiento y desarrollo. ${ }^{5}$

Considerando las etapas de desequilibrio, traslado y estabilización que propone Lomnitz ${ }^{6}$ como fundamentales en la acomodación de la familia dentro del nuevo nicho ecológico, el hecho de moverse de su lugar de origen y el riesgo de exponer al prescolar a la carencia de nutrimentos y micronutrimentos en su dieta habitual son trascendentales, pues dan lugar a la aparición de la desnutrición. La importancia de este hecho radica en el panorama que presenta la condición nutricia y alimentaria de un grupo de niños que ha estado sujeto a situaciones de tipo alimentario hostiles y, en forma indirecta, determina la disponibilidad, accesibilidad y aprovechamiento de los alimentos, cuyo resultado deja ver las condiciones socioeconómicas y de salud en una población.

En el campo de la investigación epidemiológica de la desnutrición en niños prescolares hijos de familias migrantes, son escasos los trabajos en donde se reconoce el papel que desempeña este fenómeno demográfico como una determinante de la salud. En ese contexto es relevante analizar la migración y sus repercusiones en el estado nutricio de estos niños, de tal manera que el objetivo de este estudio fue comparar la prevalencia de desnutrición entre prescolares hijos de familias migrantes y no migrantes.

\section{Material y métodos}

Se realizó un estudio transversal comparativo en población prescolar de entre 1 y 6 años adscrita a 19 parvularios de la Asociación Cáritas de Monterrey, A.C., ${ }^{*}$ durante los meses de agosto a octubre de 1996 ( $\mathrm{n}=$ 160). La ubicación geográfica de estos parvularios y de las viviendas de los prescolares estudiados está clasificada como de muy alta marginación según el Consejo Nacional de Población. ${ }^{7}$ Se excluyó a los niños con malformaciones congénitas o incluidos en algún programa de intervención en nutrición. Se reemplazó a 10 infantes (6.2\%) debido a que no se localizó a los jefes de familia para aplicar la cédula de entrevista.

Para la selección de la muestra, en una primera etapa se convocó a las representantes de los parvularios a una reunión con el fin de invitarlas a participar en el proyecto y solicitar su apoyo para la identificación de familias migrantes y no migrantes. Para esta última tarea, se aplicó una cédula filtro que incluía preguntas clave sobre migración familiar, definida ésta como el desplazamiento del jefe de familia a esa zona geográfica del estado de Nuevo León durante un periodo menor a seis años. En una segunda etapa, se seleccionó en forma aleatoria simple a la familias de los estratos migrantes y no migrantes previamente registrados. Finalmente, se incluyó aquel miembro de menor edad de la familia elegida y, en caso de gemelos, se incluyó a los dos. Si por algún motivo no se localizaba a la familia seleccionada, se tomaba la siguiente en la lista.

El tamaño de la muestra se calculó con base en la fórmula para estimar diferencia de proporciones, ${ }^{8}$ considerando una $p_{1}=.50$ de desnutridos en familias migrantes y una $p_{2}=.30$ en familias no migrantes (proporciones estimadas en una prueba piloto) Se empleó un margen de error de $5 \%$ y un intervalo de confianza (IC) de 95\%. El resultado indicó que se requerían por lo menos 57 prescolares de familias migrantes y 57 de familias no migrantes.

Se diseñó un cuestionario estructurado que tendría que aplicarse mediante entrevista con el jefe de la familia del prescolar en estudio. La cédula contenía información sobre características demográficas del prescolar, como lugar de nacimiento y fecha del último cumpleaños, así como las medidas antropométricas

\footnotetext{
* Asociación civil no lucrativa cuya misión es auxiliar a la población más desprotegida en la solución de problemas individuales y comunitarios.
} 
de peso y talla; también incluía características socioeconómicas familiares como ocupación, ingreso y escolaridad del jefe de familia. El ingreso se clasificó de acuerdo con el salario mínimo percibido a la semana (región norte), según la Comisión Nacional de Salarios Mínimos. En cuanto a la escolaridad del jefe de familia se consideraron los años de haber recibido instrucción educativa y se agrupó en jefes sin escolaridad, con primaria incompleta, primaria completa y superior a la primaria. En lo referente a la historia migratoria, se registró: a) lugar de origen del jefe de familia; b) marginación del lugar de origen, la cual se clasificó, según el Consejo Nacional de Población ${ }^{7}$ y con base en nueve indicadores socioeconómicos, en grados: muy baja, baja, media, alta y muy alta; c) dirección de desplazamiento: rural-urbano y urbano-urbano, y d) intensidad del movimiento en los últimos 12 meses $(0=$ no movimiento), agrupado en categorías de acuerdo con las veces que salió y regresó a su lugar de origen, el tiempo de haber migrado y el motivo ecológico por el que se movió, así como el socioeconómico, es decir, la falta de trabajo en el lugar de origen, el acceso a vivienda y alimento, y otros como el haber acompañado a familiares.

Antes de llevar a cabo la evaluación antropométrica puntual, se capacitó al personal participante con la finalidad de estandarizar los criterios para tomar las medidas antropométricas, en relación con los métodos, técnicas y procedimientos que se emplearían, de acuerdo con lo recomendado por el Centro de Capacitación Integral para Promotores Comunitarios del Instituto Nacional de la Nutrición Salvador Zubirán, con el fin de recolectar los datos que permitieran su replicabilidad y minimizar los errores entre las distintas mediciones. ${ }^{9}$ Dos personas se encargaron de tomar las medidas antropométricas: una de ellas tomó el peso y la talla del niño, y la otra anotó los datos. Para determinar el peso de los niños se utilizó la báscula con estadímetro en centímetros y precisión de hasta $100 \mathrm{~g}$ marca Detecto con balance máximo de $140 \mathrm{~kg}$. Para determinar la talla se utilizó la báscula de pedestal o el infantómetro, según correspondiera.

Para evaluar el estado nutricio del niño se consideraron los indicadores nutricionales peso/talla y talla/edad recomendados por la Organización Mundial de la Salud (OMS). ${ }^{10}$ El punto de corte para establecer la desnutrición fue en unidades de desviaciones estándar (DE) (puntaje Z); se consideró desnutrición cuando los indicadores se ubicaron en -2 DE por debajo de la mediana de la población de referencia.

El plan de análisis incluyó estimación de prevalencias, razones de prevalencia (RP), intervalos de confianza (IC) de $95 \%$ y puntajes $\mathrm{Z}$.

\section{Resultados}

La edad promedio de la población en estudio correspondió a $56.2 \pm 13.5$ meses; $52.5 \%$ pertenecía al sexo masculino y $59.4 \%$ provenía de familias cuyo jefe tenía una ocupación no calificada, con un ingreso semanal promedio de $214 \pm 68.4$ pesos. De los jefes de familia, 42.5\% poseía una educación que iba más allá de la primaria; $27.5 \%$ tenía primaria incompleta; $25 \%$, primaria completa, y $5 \%$ no tenía ninguna escolaridad.

Entre los prescolares, 26.2\% tenía el antecedente de haber nacido fuera del estado de Nuevo León. De los jefes de familia, $52.8 \%$ refirió el antecedente de migración en los últimos seis años; el motivo ecológico predominante de la migración fue "la ausencia de trabajo" $(48.8 \%)$, seguido por el "deseo de mejorar el nivel de vida" (24\%), "acompañante de pariente" $(20 \%) \mathrm{y}$, porque "lo trajeron" (7.2\%). En el cuadro I se observan con detalle las características migratorias.

\section{Cuadro I \\ Características migratorias del jefe de familia DE PREsColares (1 a 6 años). MonterRey, Nuevo León, México, Agosto a octubre de 1996}

$\begin{array}{cc}\text { Característica migratoria } & \mathrm{n}=80 \\ \%\end{array}$

- Grado de marginación en el lugar de origen

\begin{tabular}{lr} 
Muy baja & 7.5 \\
\hline Baja & 25.0 \\
\hline Media & 12.5 \\
\hline Alta & 50.0 \\
\hline Muy alta & 5.0
\end{tabular}

- Dirección del desplazamiento

Rural-urbano $\quad 67.5$

Urbano-urbano

32.5

- Intensidad del movimiento en el último año

\begin{tabular}{lr}
0 & 98.7 \\
\hline $1-2$ & 1.3
\end{tabular}

- Temporalidad de la migración

\begin{tabular}{lc} 
Definitivo & 97.5 \\
\hline Pendular & 1.3 \\
\hline Sin definición & 1.2
\end{tabular}

- Tiempo de haber migrado (meses)

\begin{tabular}{lr}
$\leq 24$ & 6.3 \\
\hline $25-71$ & 93.7
\end{tabular} 
La media de peso observada fue de $14.3 \pm 2.4 \mathrm{~kg}$, y la media de talla, de $100.8 \pm 8.8 \mathrm{~cm}$. La prevalencia de desnutrición según el indicador talla/edad correspondió a 40 por 100 prescolares y, según el indicador peso/talla, a 16.8\%. La prevalencia de desnutrición en prescolares de familias migrantes fue de $51.3 \%$ en comparación con la de no migrantes, que fue de $28.8 \%$ $(\mathrm{RP}=2.6$, IC 95\% $=1.2,5.2, p=0.006)$.

El análisis univariado mostró que ninguna característica socioeconómica influyó sobre el estado nutricional (cuadro II); sólo las migratorias como lugar de origen, grado de marginación y dirección del desplazamiento influyen (cuadros II y III). En el análisis estratificado se observó que el antecedente de migración incrementó el riesgo en los prescolares del sexo femenino, en familias con jefes con ocupación no calificada, con ingreso menor o igual a dos salarios mínimos y de escolaridad primaria completa y más (cuadro IV).

En el cuadro V se observa la prevalencia de desnutrición por puntajes $\mathrm{Z}$, grupos de edad e indicador nutricional.

\section{Discusión}

La población de prescolares estudiada constaba de niños y niñas, en proporción similar, con una edad promedio de cinco años. Se ha mencionado que la edad de mayor riesgo nutricional se encuentra entre el primer y segundo año de vida. ${ }^{11}$ Sin embargo, en este estudio hubo un número reducido disponible de niños con esta edad, en la comunidad de prescolares atendidos en las unidades educativas de la Asociación Cáritas. Los niños que dicha asociación acepta son los que por alguna causa, entre ellas la migración de la familia, no logran ingresar a escuelas oficiales, razón por la cual predominan las edades mencionadas. Sin embargo, esa limitante no impide analizar el fenómeno de la desnutrición, ya que estos grupos de edad no deben quedar al margen de las investigaciones. En el Censo Nacional de Talla de 1993, en escolares de primer grado de primaria se observó una prevalencia de $20 \%$ de desnutrición a la edad de seis años y de $40 \%$ en aquellos de nueve años, sobre todo en las zonas que

\section{Cuadro II}

\section{RELACIÓN DE CARACTERISTICAS SOCIODEMOGRÁFICAS Y ESTADO NUTRICIONAL, SEGÚN INDICADOR TALLA/EDAD.} Monterrey, Nuevo León, México, agosto a octubre de 1996

\begin{tabular}{|c|c|c|c|c|}
\hline \multirow{3}{*}{ Variable } & \multicolumn{2}{|c|}{ Desnutrición } & \multirow{3}{*}{$\mathrm{RP}$} & \multirow[b]{2}{*}{ IC $95 \%$} \\
\hline & Sí $(n=64)$ & No $(n=96)$ & & \\
\hline & $\%$ & $\%$ & & \\
\hline \multicolumn{5}{|l|}{ - Edad (meses) } \\
\hline $12-35$ & 6.2 & 14.5 & 1.00 & $.20,4.8$ \\
\hline $36-47$ & 4.6 & 6.3 & 0.56 & $.10,2.8$ \\
\hline $48-59$ & 33.0 & 29.2 & 0.50 & $.06,3.2$ \\
\hline \multirow[t]{2}{*}{$60-71$} & 56.2 & 50.0 & 1.00 & \\
\hline & & & $\mathrm{MH} .68$ & $.26,1.71$ \\
\hline \multicolumn{5}{|l|}{ - Sexo } \\
\hline Masculino & 54.7 & 51.0 & 1.10 & $.58,2.2$ \\
\hline Femenino & 45.3 & 49.0 & & \\
\hline \multicolumn{5}{|l|}{ - 0 cupación del jefe familia } \\
\hline Calificado & 37.5 & 42.7 & 0.80 & $.40,1.6$ \\
\hline No calificado & 62.5 & 57.3 & & \\
\hline \multicolumn{5}{|l|}{ - Ingreso del jefe familia } \\
\hline$\leq 2$ salarios mínimos & 85.9 & 85.4 & 1.40 & $.39,2.8$ \\
\hline >2 salarios mínimos & 14.1 & 14.6 & & \\
\hline \multicolumn{5}{|l|}{ - Escolaridad del jefe familia } \\
\hline Primaria o menos & 35.9 & 30.2 & 1.30 & $.63,2.6$ \\
\hline Más de primaria & 64.1 & 69.8 & & \\
\hline $\mathrm{RP}=$ razón de prevalencia & Haenzel & & & \\
\hline
\end{tabular}




\section{Cuadro III \\ ReLACIÓN DE CARACTERÍSTICAS MIGRATORIAS Y ESTADO NUTRICIONAL, SEGÚN INDICADOR TALLA/EDAD. Monterrey, Nuevo León México, agosto a octubre de 1996}

\begin{tabular}{|c|c|c|c|c|}
\hline \multirow{3}{*}{ Variable } & \multicolumn{2}{|c|}{ Desnutrición } & \multirow[b]{2}{*}{$\mathrm{RP}$} & \multirow[b]{2}{*}{ IC $95 \%$} \\
\hline & Sí $(n=64)$ & No $(n=96)$ & & \\
\hline & $\%$ & $\%$ & & \\
\hline - Lugar de origen & & & 1.0 & \\
\hline N uevo León & 43.8 & 62.5 & 3.2 & $1.3,7.5^{*}$ \\
\hline San Luis Potosí y Tamaulipas & 37.5 & 16.7 & 0.92 & $.82,11.64$ \\
\hline Zacatecas y C oahuila & 9.4 & 14.6 & 2.1 & $.31,6.2$ \\
\hline 0 tros estados & 9.4 & 6.2 & MH 2.1 & $1.1,2.6^{\ddagger}$ \\
\hline \multicolumn{5}{|c|}{ - Grado de marginación lugar de origen } \\
\hline No migración & 35.9 & 58.3 & 1.0 & \\
\hline Muy baja y baja & 25.0 & 10.4 & 3.9 & $1.4,10.9 \neq$ \\
\hline Media & 9.4 & 4.2 & 3.6 & $.81,17.3$ \\
\hline Alta y muy alta & 29.7 & 26.0 & 1.8 & $.80,4.2$ \\
\hline & & & MH 2.6 & $1.5,4.7^{\ddagger}$ \\
\hline \multicolumn{5}{|l|}{ - Dirección de desplazamiento } \\
\hline No migración & 35.9 & 59.4 & 1.0 & \\
\hline Urbano-urbano & 23.4 & 11.5 & 3.3 & $1.2,9.3^{*}$ \\
\hline Rural-urbano & 40.7 & 29.2 & 2.3 & $1.05,5.0 *$ \\
\hline $\begin{array}{l}\mathrm{RP}=\text { razón de prevalencia } \\
\mathrm{MH}=\text { Mantel Haenzel }\end{array}$ & $\begin{array}{l}* p \leq 04 \\
{ }^{*} p \leq .01\end{array}$ & & MH 2.6 & $1.4,4.9 *$ \\
\hline
\end{tabular}

ya están clasificadas como de alto riesgo nutricional, en los estados del sureste. ${ }^{12}$

La prevalencia de desnutrición fue casi semejante a la del 30\% que se registró en la zona norte del país, según el indicador talla para la edad; ${ }^{2}$ su distribución fue similar por sexo, grupo de edad y características socioeconómicas del jefe de la familia, y ninguno de estos factores incrementó el riesgo por desnutrición a nivel univariado. No obstante, en diversos estudios se ha mencionado el ingreso como una variable que determina directamente la condición nutricional de los prescolares ${ }^{13,14}$ y se ha llegado a la conclusión de que las comunidades donde prevalece un nivel socioeconómico bajo, la situación nutricional es deficiente. ${ }^{5,6,15,16}$ Es probable que en este estudio ese factor no haya dado lugar a un efecto negativo porque la población estudiada era afín respecto a la frecuencia de esta característica entre los grupos de desnutridos y no desnutridos que atiende la Asociación; como ya se mencionó, ésta se limita a los niños de escasos recursos socioeconómicos. Precisamente se ubicó a esta población por constituir una comunidad en riesgo nutricional potencial.
La prevalencia de desnutrición, de acuerdo con el indicador talla para la edad, se incrementó casi al doble en los niños pertenecientes a familias migrantes, lo cual es similar a los hallazgos de Salcedo y Prado, ${ }^{15}$ quienes registraron una prevalencia de $68 \%$ en migrantes cañeros de Jalisco. Estas prevalencias sugieren la carencia prolongada de los nutrimentos necesarios en esta etapa de la vida, de tal manera que el riesgo de morbilidad y mortalidad aumenta en estos grupos. ${ }^{11,17,18}$ Más de la mitad de los jefes de familia presentaron el antecedente de haber migrado desde áreas rurales con zonas de marginación media, alta y muy alta. El motivo ecológico más frecuente de la migración fue el de orden social y económico; sin embargo, estas personas continúan padeciendo condiciones socioeconómicas poco favorables, confirmando así la teoría de Winnie ${ }^{19} \mathrm{en} \mathrm{su}$ estudio sobre movilidad demográfica en el estado de Jalisco.

Los lugares de origen de la migración como San Luis Potosí y Tamaulipas incrementaron 3.2 veces el riesgo de desnutrición para el indicador talla/edad en comparación con aquellos de Nuevo León o con los no migrantes. Con referencia a este factor, la Encuesta Na- 


\section{Cuadro IV \\ ReLACIÓN DE LA MIGRACIÓN CON DESNUTRICIÓN ESTRATIFICADA POR CARACTERÍSTICAS Socioeconómicas. Monterrey, Nuevo León, México, AGosto A oCtUBRe de 1996}

\begin{tabular}{llc} 
Variable & RP & IC $95 \%$ \\
Edad prescolar 48-59 meses & 0.91 & $.25,3.2$ \\
\hline Edad prescolar 60-71 meses & 3.0 & $1.1,8.3^{\ddagger}$ \\
\hline Sexo masculino del prescolar & 1.5 & $.63,4.0$ \\
\hline Sexo femenino del prescolar & 2.9 & $1.0,9.1^{*}$ \\
\hline O cupación calificada del jefe familia & 1.9 & $.61,6.1$ \\
\hline O cupación no calificada del jefe familia & 3.1 & $1.2,8.3^{*}$ \\
\hline Ingreso $\leq 2$ salarios mínimos del jefe de familia & 2.4 & $1.1,5.1^{*}$ \\
\hline Ingreso >2 salarios mínimos del jefe de familia & 4.6 & $.53,49.5$ \\
\hline Escolaridad ninguna y primaria incompleta & 1.5 & $.43,5.4$ \\
\hline Escolaridad primaria completa y más & 3.3 & $1.3,8.2^{*}$ \\
\hline
\end{tabular}

$\mathrm{RP}=$ razón de prevalencia

${ }^{*} p \leq .03 \quad{ }^{\ddagger} p \leq .01$

N ota: no se observaron prescolares desnutridos en no migrantes en los grupos de edad de 12 a 35 y 36 a 47 meses cional de Nutrición de 1988 reveló un mayor riesgo de desnutrición en niños prescolares de poblaciones rural e indígena. ${ }^{17}$ Asimismo, la Encuesta Nacional de Alimentación en el Medio Rural de $1989^{20}$ identificó las zonas centro-norte y noreste como nutricionalmente afectadas, donde quedan incluidos los estados de Zacatecas, San Luis Potosí y Tamaulipas. En relación con el tiempo de migración, en este estudio se consideró como máximo seis años de migración, ya que después de este lapso la familia migrante se inserta al proceso productivo y, por lo tanto, presenta un menor riesgo en comparación con periodos menores de migración. En los primeros años se presentan cambios biopsicosocioculturales, incluyendo el rechazo inicial y el acomodo provisorio, lo cual tiene como consecuencia un desequilibrio en la atención nutricia del prescolar. ${ }^{6}$

El análisis estratificado mostró diferencias evidentes entre familias migrantes y no migrantes; la migración incrementó tres veces el riesgo para desnutrición en los niños de 60 a 71 meses; no obstante, este efecto no se mantuvo para aquellos de entre 48 y 59 meses. Desafortunadamente, no se pudo analizar esta información en los infantes de 36 a 47 meses y de menos

\section{Cuadro V \\ Prevalencia de desnutrición y puntajes $Z$ por grupos de edad según indicador nUtricional y antecedente de migración. Monterrey, Nuevo León, México, agosto a octubre de 1996}

\begin{tabular}{|c|c|c|c|}
\hline \multirow[b]{2}{*}{ Indicador por grupo de edad } & \multicolumn{2}{|c|}{$\begin{array}{l}\text { M igrantes } \\
\text { Puntajes Z }\end{array}$} & $\begin{array}{l}\text { No migrantes } \\
\text { Puntajes Z }\end{array}$ \\
\hline & $\begin{array}{l}P \\
\%\end{array}$ & DE & $\begin{array}{l}P \\
\%\end{array}$ \\
\hline
\end{tabular}

\begin{tabular}{rlllll} 
12-35 meses & & & & & - \\
Talla/edad & 80.0 & -2.2 & .45 & 0 & - \\
\hline Peso/talla & 20.0 & -2.6 & .41 & 0 & -
\end{tabular}

\begin{tabular}{lllllll} 
36-47 meses & & & & & \\
Talla/edad & 37.5 & -2.8 & .42 & 0 & - \\
\hline Peso/talla & 10.0 & -2.4 & 0 & 0 & -
\end{tabular}

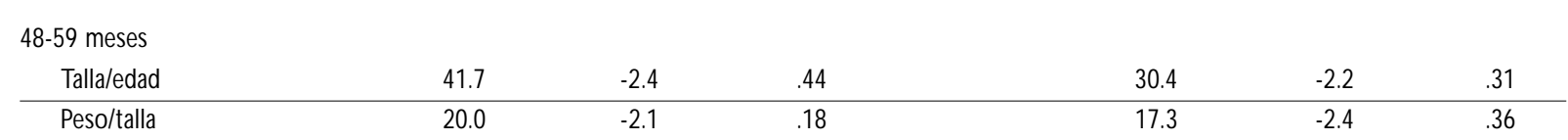

\begin{tabular}{lllllll}
$\begin{array}{l}\text { 60-71 meses } \\
\text { Talla/edad }\end{array}$ & 55.8 & -2.4 & .41 & 32.5 & -2.3 & .36 \\
\hline Peso/talla & 16.3 & -2.5 & .41 & 25.0 & -2.4 & .36
\end{tabular}

\begin{tabular}{|c|c|c|c|c|c|c|}
\hline \multicolumn{7}{|l|}{ Total } \\
\hline Talla/edad & 51.3 & -2.4 & .40 & 28.8 & -2.3 & .33 \\
\hline Peso/ta & 13.8 & -2.4 & .37 & 13.7 & -2.2 & .46 \\
\hline
\end{tabular}

$P=$ prevalencia

$D E=$ desviación estándar 
de 36 meses de edad, por la ausencia de infantes desnutridos en el grupo de no migrantes. Salcedo y Prado ${ }^{15}$ registraron la prevalencia más baja de desnutrición en infantes con edades de 60 a 71 meses, y la más alta en aquellos de 12 a 23 meses ( 71 y $82 \%$, respectivamente). La mayoría procedía de comunidades indígenas y presentaba una temporalidad de la migración tipo pendular, es decir, regresaba a su lugar de origen una vez concluido su trabajo en el lugar de destino. Ysunza y colaboradores ${ }^{21}$ identificaron desnutrición con déficit de talla casi en $40 \%$ de los prescolares migrantes estudiados, en grupos de edad de 12 a 24 meses y de 25 a 59 meses.

La migración también representó un factor de riesgo de desnutrición en prescolares del sexo femenino, pertenecientes a familias donde el padre tenía una ocupación no calificada y un ingreso semanal menor o igual a los dos salarios mínimos. Una particularidad común a todas las familias migrantes es su inserción en el mercado laboral mal remunerado. En su trabajo Salcedo y Prado ${ }^{15}$ observaron un incremento en el riesgo de desnutrición en los niños de familias donde el padre carecía de escolaridad en comparación con aquellos que habían cursado la primaria o más años de instrucción. Sin embargo, en este estudio se observó influencia de la escolaridad de primaria completa y más de los jefes de familia migrantes sobre el estado nutricional de sus hijos; una explicación podría ser que la mayor parte de ellos tenía ese nivel de escolaridad.

En función de los puntajes Z, el grupo de prescolares de familias migrantes que tenían mayor prioridad para ser sometidos a una intervención nutricional fue el de 36 a 47 meses de edad, con base en el indicador talla/edad, y el de 12 a 35 meses, considerando el indicador peso/talla; el resto de los infantes presentaron prioridades similares.

La salud y la nutrición adecuada se logran mediante una alimentación y condiciones socioambientales buenas. Es indudable que las condiciones socioeconómicas actuales deben mejorar; mientras tanto, es necesario detectar aquellas poblaciones que se encuentran en riesgo de padecer desnutrición y establecer a la brevedad medidas de intervención en nutrición, al igual que sucedió en el grupo de hijos de familias migrantes que se presenta en este estudio. No sólo es conveniente sino también trascendente considerar los procesos demográficos como elementos clave en la planeación de los servicios de salud, especialmente los relacionados con la nutrición.

Un punto importante es el relacionado con los indicadores peso/talla y talla/edad que se utilizaron para determinar la condición nutricia, y que se eligieron en esta investigación para hacer hallazgos compa- rables con estudios semejantes. El patrón de crecimiento se evaluó comparando los indicadores talla/ edad observados con aquellos registrados como ideales por el Centro Nacional de Estadísticas en Salud de Estados Unidos (National Center for Health Statistics, NCHS) y recomendados por la Organización Mundial de la Salud, los cuales están basados en niños estadunidenses. En la actualidad se sabe que, por lo menos hasta los 10 años de edad, los niños de cualquier raza tienen el mismo potencial de crecimiento, siempre y cuando existan las condiciones nutricionales y ambientales adecuadas. Lo anterior justifica la utilización de un patrón extranjero en la evaluación de estos niños. ${ }^{22}$

Los resultados de este estudio pueden extrapolarse a poblaciones con características semejantes. Es necesario realizar investigaciones retrospectivas o prospectivas para conocer la situación real de la población general y con otras características de migración tales como la de desplazamiento rural-urbano, rural-internacional y urbano-internacional, para obtener pruebas concluyentes que determinen la asociación causaefecto del proceso salud-enfermedad. Es importante incorporar las variables sociales a las investigaciones en nutrición; si bien su abordaje teórico y metodológico no es fácil, se deben considerar de manera interdisciplinaria en este proceso.

\section{Recomendaciones}

- La prevalencia de la desnutrición crónica en este grupo de riesgo justifica la prioridad de establecer estrategias de intervención alimentaria, principalmente ayuda alimentaria directa a estos niños y tomar en cuenta las migraciones en la planeación en salud.

- Se propone establecer un sistema de vigilancia nutricional con indicadores antropométricos y socioeconómicos para detectar grupos sujetos a riesgo e intervenir de manera precoz y oportuna en los casos de desnutrición. Asimismo, es recomendable instituir un sistema de capacitación en el área de alimentación y nutrición para el personal encargado de los parvularios, con el fin de que, a su vez, como agentes multiplicadores, lo transmitan a los padres de familia de los prescolares.

\section{Agradecimientos}

Las autoras agradecen a la doctora Ana María Salinas por su invaluable asesoría; asimismo, al personal administrativo y voluntario del Programa Parvularios de la Asociación Cáritas de Monterrey, A.C. 


\section{Referencias}

1. Jelliffe-D errick B. N utrición infantil en países en desarrollo. 3a. edición. México, D.F.: Editorial Limusa, 1974.

2. Dirección General de Epidemiología, Secretaría de Salud. Encuesta N acional de Nutrición. México, D.F.: SSA, 1988.

3. Dirección General de Estadística, Informática y Evaluación, Secretaría de Salud. Mortalidad 1993. México, D.F.: SSA, 1993.

4. Instituto N acional de Estadística Geografía e Informática. Encuesta N acional de la Dinámica Demográfica. A guascalientes: IN EGI, 1992.

5. Torres-Pérez W. Componentes del comportamiento social humano en la desnutrición del niño campesino. Rev Inst N ac N utr Salvador Zubirán 1992;3:13-16.

6. Lomnitz L. Cómo sobreviven los marginados. 12a. edición. México, D.F.: Editorial Siglo XXI, 1993.

7. Instituto $\mathrm{N}$ acional de Estadística G eografía e Informática. Consejo $\mathrm{N}$ acional de Población. Indice y grado de marginación de cada entidad federativa, lugar que ocupa en el contexto estatal y nacional. México, D.F.: Consejo Nacional de Población/Comisión N acional del Agua, 1993.

8. Salinas AM, Villarreal E, Garza ME. La investigación en ciencias de la salud, una actividad sencilla. Monterrey, N .L.: Universidad Autónoma de Nuevo León, 1996.

9. Peláez ML,Torre P, Ysunza A. Elementos prácticos para el diagnóstico de la desnutrición. Centro de capacitación integral para promotores comunitarios. México, D.F.: Instituto $\mathrm{N}$ acional de la Nutrición Salvador Zubirán, 1993.

10. O rganización Mundial de la Salud. Medición del cambio del estado nutricional. Ginebra: O MS, 1983.

11. A vila A. Hambre, desnutrición y sociedad. La investigación epidemiológica de la desnutrición en México. Guadalajara, Jal.:U Universidad de Guadalajara, 1990.
12. Sistema N acional para el Desarrollo Integral de la Familia (DIF). Censo $\mathrm{N}$ acional de Talla 1993. México, D.F. : DIF, 1994.

13. Pérez-Gil S. La influencia de factores sociales sobre la situación nutricional de preescolares en una área marginada. Rev Invest Clin 1986;38: 83-95.

14. Victora C, Barros F, Vaughan J. Epidemiología de la desigualdad. San Pablo, Brazil: Editora de Humanismo, C Ciencia y Tecnología, 1992.

15. Salcedo-Rocha A, Prado-A guilar C. El proceso migratorio como factor de riesgo en la desnutrición crónica del niño preescolar migrante cañero en Jalisco. Salud Publica Mex 1992;34:518-522.

16. Madrigal H, Guarneros N, Pérez-Gil S, Moreno-Terraza 0, Romero J. Programa integrado para la atención nutricional. Una experiencia metodológica en el estado de San Luis Potosí. Rev Inst $\mathrm{N}$ ac N utr Salvador Zubirán 1992;3:9-17.

17. Rivera-D ommarco J, González-Cossío T, Flores M, Hernández-Avila M, Lezana MA, Sepúlveda-A mor J. Déficit de talla y emaciación en menores de cinco años en distintas regiones y estratos en México. Salud Publica Mex 1995;37:95-107.

18. Martorell R, Ho TJ. Malnutrition, morbidity and mortality. Popul Dev Rev 1984;10 suppl:49-68.

19. W innie W. Movilidad demográfica y su incidencia en una región de fuerte emigración. El caso de 0 ccidente. Guadalajara, Jal.: U niversidad de Guadalajara, 1984.

20. División N utrición de Comunidad, Instituto $\mathrm{N}$ acional de la $\mathrm{N}$ utrición Salvador Zubirán. Encuesta $N$ acional de Alimentación en el Medio Rural, 1989. México, D.F.: IN N SZ, 1990.

21.Y sunza A, C oello MP, Pérez SE, Baz G, Hernández M, Arana M. D ietas de transición y riesgo nutricional en población migratoria. México,D.F.: Instituto N acional de la Nutrición Salvador Zubirán, 1985:17-19.

22. Habicht JP, Martorell R. Height and weight standards for pre-school children. How relevant are ethnic differences in growth potential? Lancet 1974;1:611. 